\title{
INT-777 Improves Cognitive Impairment after Sepsis by Attenuating Neuroinflammation Through the TGR5/cAMP/PKA/CREB Signaling Axis in Rats
}

Peng Jin

Huashan Hospital Fudan University

Shuixiang Deng

Huashan Hospital Fudan University

Mi Tian

Huashan Hospital Fudan University

Pengju Wei

Fudan University

Yao Wang

Huashan Hospital Fudan University

Jiaying Tan

Huashan Hospital Fudan University

Huimei Wen

Huashan Hospital Fudan University

Feng Zhao

Huashan Hospital Fudan University

Yanqin Gao

Fudan University

YE GONG ( $\nabla$ gong_ye@fudan.edu.cn )

Huashan Hospital Fudan University

\section{Research}

Keywords: sepsis-associated encephalopathy, TGR5, INT-777, cognitive impairment, Neuroprotection, neuroinflammation

Posted Date: June 29th, 2020

DOI: https://doi.org/10.21203/rs.3.rs-36666/v1

License: (a) (i) This work is licensed under a Creative Commons Attribution 4.0 International License.

Read Full License 


\section{Abstract}

Background: Sepsis survivors are left with significant cognitive and behavioral impairments after discharge, but research on the relevant mechanisms and interventions remains lacking. TGR5 plays a neuroprotective role in many neurologic disease models through different mechanisms. To date, no studies have assessed the effects of TGR5 on neuroinflammation or cognitive or behavioral changes in sepsis models.

Methods: A total of 267 eight-week-old male Sprague-Dawley rats were used in this study. Sepsis was induced by cecal ligation and puncture (CLP). All animals received volume resuscitation. The rats were given TGR5 CRISPR oligonucleotide intracerebroventricularly 48 hours before CLP surgery. INT-777 was administered intranasally 1 hour after CLP, and the CAMP inhibitor SQ22536 was administered intracerebroventricularly 1 hour after CLP. Survival rate, bodyweight change, clinical score, neurobehavioral tests, western blot, and immunofluorescence staining were performed. The cognitive function of rats was measured by Morris water maze during 15-20 days after CLP.

Results: The expression of TGR5 in the rat hippocampus was upregulated and peaked at 3 days after CLP. The survival rate of rats after CLP was less than $50 \%$, and the growth rate in terms of weight was significantly decreased, while these changes were not improved by INT-777 treatment. However, INT-777 treatment reduced the clinical scores of rats at 24 hours after CLP. On day 15 and later, the surviving mice completed a series of behavioral tests. CLP rats showed spatial and memory deficits and anxiety-like behaviors, and INT-777 treatment significantly improved these effects. Mechanistically, immunofluorescence analysis showed that INT-777 treatment reduced the number of microglia in the hippocampus, neutrophil infiltration and the expression of inflammatory factors after CLP in rats. Moreover, INT-777 treatment significantly increased the expression of TGR5, cAMP, p-PKA, and p-CREB and downregulated the expression of IL-1 $\beta$, IL- 6 and TNF-a. CRISPR-mediated TGR5 knockdown and SQ22536 treatment abolished the neuroprotective effects of TGR5 activation after CLP.

Conclusion: This study demonstrates that INT-777 treatment reduced neuroinflammation and microglial cell activation, and then improved cognitive impairment in the experimental sepsis rats. TGR5 has translational potential as a therapeutic target to improve neurological outcomes in sepsis survivors.

\section{Introduction}

Sepsis is one of the leading causes of death in intensive care units worldwide and can cause multiple organ dysfunction, including neurological dysfunction (sepsis-associated encephalopathy, SAE) [1]. Sepsis associated encephalopathy is one of the most common complications among survivors of sepsis in the acute and late stages. Some studies suggest that approximately $45 \%$ of sepsis survivors develop symptoms of long-term cognitive impairment, including the impairment of memory, attention, verbal fluency, and executive function, upon discharge from the hospital, leading to a significant decline in quality of life[2, 3]. As the morbidity and mortality of sepsis increase year by year, the number of sepsis 
survivors increases steadily, and the long-term cognitive impairment of sepsis survivors becomes increasingly prominent $[4,5]$. Therefore, it is particularly important to fully understand the pathophysiological mechanism of sepsis encephalopathy and to provide effective intervention measures as soon as possible. Because the pathophysiological changes of brain dysfunction after sepsis are multifactorial and highly complex, the pathogenesis of sepsis still needs to be further elucidated. However, increasing evidence suggests that neuroinflammation, oxidative stress and BBB destruction are potential mechanisms of the brain damage and long-term cognitive impairment caused by sepsis[6-8].

G-protein-coupled bile acid receptor (Gpbar1/TGR5), is a member of the GPCR family, Since its discovery in 2002, TGR5 has been found to be commonly expressed in various tissues in humans and animals, such as the small intestine, liver, spleen, and lung, and to activate various intracellular signal transduction pathways by interacting with bile acids $[9,10]$. Recent studies have shown that in the brain, TGR5 is expressed in microglia, astrocytes, neurons, and endothelial cells[11, 12]. Activation of TGR5 can treat liver disease, kidney disease and metabolic disease through its anti-inflammatory, antiapoptotic, and antioxidative stress effects and other effects $[13,14]$. Among neurological diseases, activation of TGR5 has been found to play a neuroprotective role in subarachnoid hemorrhage, middle cerebral artery occlusion and hepatic encephalopathy in animal models[11, 12,15]. A recent study showed that activating TGR5 suppressed neuroinflammation and improved cognitive function in mice with Alzheimer's disease[16]. However, no study has evaluated the role of TGR5 in neurological impairment after sepsis.

After TGR5 was activated by the endogenous ligand bile acids, the expression of intracellular cAMP increased significantly[17]. In pancreatic $\beta$ cells, after selective activation of TGR5 by INT-777, Gas was activated, and the intracellular cAMP level then increased[18]. Activation of TGR5 to exert antiinflammatory effects is mainly achieved through the cAMP-PKA axis[19]. PKA can promote the phosphorylation of CREB in its transcription-activating site, which further promotes the binding of CREB to the transcription coactivator CBP and the formation of complexes, thereby blocking the transcription of inflammatory genes[20].

In this present study, we explored the role of TGR5 in neuroprotective after CLP. The results showed that the TGR5 activation by INT-777 improves cognitive impairment by attenuating neuroinflammation after CLP in rats at least partly though the CAMP/PKA/CREB signaling pathway.

\section{Methods}

\subsection{Animals}

A total of 267 adult Sprague-Dawley rats (weight 250-300 g) were used in this experiment. The rats were housed on a 12-hour light/dark cycle at a controlled temperature and humidity and had unlimited access to food and water. All animal experiments in this study were approved by the Animal Care and Use Committee of Fudan University. 


\subsection{Sepsis induction - CLP model}

Sepsis was induced by cecal ligation and puncture (CLP) as previously described[21]. Rats were fasted eight hours before the operation, but their access to water was not limited. The animals were anesthetized by intraperitoneal injection of a mixture of ketamine $(80 \mathrm{mg} / \mathrm{kg})$ and xylazine $(10 \mathrm{mg} / \mathrm{kg})$. Then, a $3 \mathrm{~cm}$ midline laparotomy was performed, the cecum was exposed, the ileocecal region was closed with a 3.0 thread below the ileocecal region, and the cecum was perforated with a 14-point needle and gently squeezed to remove a small amount of feces. The cecum was then placed back into the abdominal cavity, and a laparotomy was performed with a 4.0 silk suture. The animals were resuscitated immediately by hypodermic injection of normal saline $(50 \mathrm{~mL} / \mathrm{kg})$. A midline laparotomy alone was performed for rats in the sham group. Meropenem ( $10 \mathrm{mg} / \mathrm{kg}$, intraperitoneal injection) was administered at 6,24 and 48 hours after surgery. If any signs of pain were observed in the rats after surgery, the animals were euthanized and removed from the experiment. To maintain consistency in the experiment, all CLP operations were performed by the same person with surgical qualifications.

\subsection{Experimental design}

In this study, all rats were randomly assigned to each experiment (Fig. 1). Our research design is shown in Fig. 1. The researchers who performed the clinical scoring and neurobehavioral test were blinded to all group information.

\section{Experiment 1}

To evaluate the time course of endogenous TGR5 expression, 87 rats were randomly divided into the 7 following groups for western blot analysis: sham, CLP 6 h, CLP 24 h, CLP 48 h, CLP 72 h, CLP 7 d, and CLP $14 \mathrm{~d}$. After excluding rats that did not meet the criteria, each group consisted of 6 rats. An additional 4 rats from each group were used for double-labeling immunofluorescence staining to assess the colocalization of TGR5 with ionized calcium binding adaptor molecule 1 (Iba-1).

\section{Experiment 2}

To evaluate the ability of INT-777 to improve cognitive impairment after sepsis, 55 rats were randomly divided into 3 groups: sham, CLP + saline, CLP + INT-777 $(0.48 \mathrm{mg} / \mathrm{kg})$. Clinical scores were determined $6 \mathrm{~h}$ and $24 \mathrm{~h}$ after CLP, and weight changes were recorded before surgery and $24 \mathrm{~h}, 48 \mathrm{~h}, 72 \mathrm{~h}, 7 \mathrm{~d}$ and 14 $\mathrm{d}$ after CLP. The elevated plus-maze test was conducted on day 14 post-CLP, and the Morris water maze test was conducted on days $15-20$ post-ICH. The exact time that each rat died was also recorded.

\section{Experiment 3}

To evaluate the effects of TGR5 activation with INT-777 administration on neuroinflammation at $24 \mathrm{~h}$ post-CLP, a total of 64 rats were randomized into 3 groups: sham, CLP + vehicle (saline) and CLP + INT$777(0.48 \mathrm{mg} / \mathrm{kg})$. Immunofluorescence staining was carried out to quantify Iba- $1, \mathrm{IL}-1 \beta$, and 
myeloperoxidase (MPO) in the hippocampal area. Western blotting was performed to quantify Iba-1, IL$1 \beta$, and MPO protein levels.

\section{Experiment 4}

To clarify the mechanism of the neuroprotective effect of TGR5 after CLP, 87 rats were randomized into 7 groups: sham, CLP + vehicle (saline), CLP + INT- 777 (0.48 mg/kg), CLP + INT-777 + scrambled CRISPR, CLP + INT-777 + TGR5 CRISPR, CLP + INT-777 + vehicle (DMSO), and CLP + INT-777 + SQ22536. The hippocampal area was collected from each brain for western blot analysis $24 \mathrm{~h}$ after CLP.

\subsection{Drug administration}

Intranasal administration of INT777 (CSNpharm, USA) at $1 \mathrm{~h}$ after CLP was performed as previously described[11]. The animals were anesthetized with $2 \%$ isoflurane and placed in the supine position, after which saline or INT777 $(0.48 \mathrm{mg} / \mathrm{kg})$ was administered intranasally over a period of $20 \mathrm{~min}$. The total volume delivered was $50 \mu$ l.

As described previously, TGR5 CRISPR and SQ22536 were administered intracerebroventricularly (i.c.v) $[12,22]$. The rats were anesthetized with isoflurane and fixed on a stereotaxic frame. Then, a $10 \mu \mathrm{l}$ syringe was inserted into the left lateral ventricle at the following coordinates $(0.9 \mathrm{~mm}$ posterior and $1.5 \mathrm{~mm}$ lateral to the bregma, $3.2 \mathrm{~mm}$ under the dura). A total of $2 \mu \mathrm{g}$ of TGR5 CRISPR knockout (Santa Cruz Biotechnology) or scrambled CRISPR (Santa Cruz Biotechnology) oligonucleotides per rat was given at a rate of $1 \mu \mathrm{l} / \mathrm{min}$ at 48 hours before CLP. An inhibitor of cAMP (SQ22536, Abcam, $200 \mathrm{ng} / 0.4 \mu \mathrm{l}$ ) was also administered intracerebroventricularly 1 hour after CLP.

\subsection{Clinical scoring}

Clinical scores for sepsis in the rats were determined based on a modified SHIRPA protocol[23]. The observed indicators were behavioral characteristics (activity, posture, response to stimuli), fur characteristics, respiratory rhythm, body temperature, fecal traits, and body weight, with the rats given a possible score of 1 for each trait, for a maximum score of 12 .

\subsection{Elevated plus-maze test}

An elevated plus-maze consisting of two open arms and a pair of closed arms was placed in a dim, enclosed room. At the beginning of the experiment, the rats were placed in the maze at the central point facing the closed arm, and their activities were recorded for 3 minutes. The observed indicators were number of open arm entries (two front legs must enter the arm), the open arm residence time, the number of closed arm entries, and the closed arm residence time. The open arm residence time, the frequency of open arm entries, and the total frequency of entries into the elevated maze were calculated.

\subsection{Morris water maze test}

To assess the spatial learning and memory abilities of the rats, a Morris water maze tested was conducted 15-19 days after CLP as previously described[24]. On days 1-4 of the experiment, the rats 
were trained. Briefly, the rats were placed in water that filled the apparatus, facing the wall of the apparatus, and allowed to swim for a maximum of 60 seconds. When the rats climbed onto a hidden platform, the experiment was over. If the rats did not find the platform during the experiment, they were transferred to the platform and allowed to stay there for 15 seconds. The rats were trained four times a day; the platform was always in a fixed position, but its starting position in each trial was random. On day 5 , the probe trial was performed. The platform was removed from the pool, and each rat was dropped into the water and allowed to swim for 60 seconds. The time spent in the target quadrant and the number of times the rats stood on the platform were recorded.

\subsection{Immunofluorescence staining}

After the rats were anesthetized with isoflurane, the thorax was opened and intracardially perfused with $4{ }^{\circ} \mathrm{C}$ PBS and $10 \%$ formalin, after which the brain was removed. After perfusion, the whole-brain tissue samples were immersed in formalin for 1 day and dehydrated in a $30 \%$ sucrose solution for 3 days. Brain tissues were cut into $10 \mu \mathrm{m}$ thick coronal sections using an OTF cryostat (Leica Microsystems, Germany). After blocking with $5 \%$ donkey serum for 2 hours at room temperature, the brain samples were incubated overnight at $4{ }^{\circ} \mathrm{C}$ with the following primary antibodies: goat anti-lba-1 (1:100, Abcam), rabbit anti-TGR5 (1:100, Abcam), rabbit anti-myeloperoxidase (MPO, 1:500, Abcam), and rabbit anti-IL-1 $\beta$ (1:100, Abcam). The samples were then incubated with the corresponding secondary antibodies (1:200) for $1 \mathrm{~h}$ at room temperature, followed by visualization.

\subsection{Nissl staining}

The procedure for Nissl staining was the same as in previous studies[25]. Brain slices (10 $\mathrm{mm}$ thick) were dehydrated in $95 \%$ and $70 \%$ FLEX (ThermoFisher) for 1 min respectively, then rinsed with tap water and distilled water for $10 \mathrm{~s}$. The brain slices were then stained in $0.5 \%$ cresyl violet (Sigma-Aldrich) for $1.5 \mathrm{~min}$, followed by rinsing with distilled water for $10 \mathrm{~s}$, and then put into the dehydration in $100 \%$ FLEX and xylene for $1 \mathrm{~min}$ (twice each). Finally, apply permount and place the coverslip. The sections were imaged using a microscope (Olympus-BX51). Calculate the average number of surviving neurons at 200x magnification within the hippocampal CA1 and estimate the loss of neuronal density.

\subsection{Western blot analysis}

After the rats were placed on deep anesthesia, the whole brains were quickly removed after intracardial perfusion with ice-cold PBS, and the hippocampus was isolated and stored at $-80{ }^{\circ} \mathrm{C}$. Proteins were extracted according to a methods used in a previous study[6]. After the protein concentration had been determined, samples containing equivalent amounts of protein were separated on SDS-PAGE gels at different concentrations $(7.5 \%, 10 \%, 12 \%)$ and transferred to nitrocellulose membranes. After blocking with $5 \%$ dry skim milk at room temperature for $1 \mathrm{~h}$, the membranes were incubated overnight at $4{ }^{\circ} \mathrm{C}$ with the following primary antibodies: anti-TGR5 (1:1000, Abcam), anti-CAMP (1:10000, Abcam), and anti-pPKA (1:200, Abcam); anti-PKA (1:1000, Abcam); anti-p-CREB (1:500, Novus Biologicals); anti-CREB (1:1000, Novus Biologicals); anti-MPO (1:1000, Abcam); anti-Iba-1 (1:1000, Abcam); anti-IL-1 $\beta$ (1:200, Abcam) and anti-IL-6 (1:1000 Santa Cruz Biotechnology, USA); anti-TNF-a (1:500, Abcam); anti-GAPDH 
(1:5000, Abcam); and anti-actin (1:2000, Santa Cruz Biotechnology). The next day, the cells were incubated with an appropriate secondary antibody (Santa Cruz Biotechnology, 1:3000) at room temperature for 2 hours. The immune complex bands were observed with the ECL Plus kit, and the membrane was then exposed to X-ray film.

\subsection{Statistical analyses}

All data are presented as the mean \pm SD and were subjected to one-way ANOVA followed by Tukey's multiple comparison test. Survival curves were derived by the Kaplan-Meier method and compared by the log-rank test. Differences for which $p<0.05$ were considered statistically significant. All tests were performed with GraphPad Prism 8.

\section{Results}

\subsection{Time course and cellular localization of endogenous TGR5 expression in the hippocampus after CLP}

To assess the cellular localization of TGR5, rats were sacrificed 24 hours after CLP, and their brains were removed for immunofluorescence analysis. The results showed that TGR5 was expressed in microglia. Furthermore, more TGR5-positive cells were visualized within the hippocampus of rats in the CLP group than in those of rats in the sham group (Fig. 2A). Double immunofluorescence staining of TGR5 with GFAP and NeuN was also performed at $24 \mathrm{~h}$ after CLP. The results showed that TGR5 were also expressed in neurons and astrocytes respectively (Supplementary figures). Western blotting was used to evaluate the expression of endogenous TGR5 at 6 h, $24 h, 48$ h, 72 h, $7 \mathrm{~d}$ and $14 \mathrm{~d}$ after CLP. The results showed that compared with TGR5 expression in the sham group, after CLP, TGR5 expression began to increase significantly at $6 \mathrm{~h}$ after CLP ( $p<0.05 \mathrm{VS}$ sham group), peaked on the 3rd day after CLP, and then decreased slowly, approaching the level of the sham group at 14 days after CLP. (Fig. 2C and D).

\subsection{Effects of INT-777 treatment on clinical score, body weight and survival rate}

A total of 267 rats were used in the whole experiment: 32 in the sham group and 234 in the CLP group. No rats in the sham group died, while 136 rats in the CLP group died, giving a mortality rate of $58.1 \%$ (136/234). To evaluate the effect of TGR5 activation, three experimental groups were set up: the sham group, CLP + vehicle group, and CLP + INT-777 group. Survival analysis showed that compared with that of the sham group, the survival rate of the CLP group was significantly reduced, with most of the deaths occurring within the first 3 days, after which the situation had basically stabilized at 6 days after CLP. However, compared with that of the CLP + vehicle group, INT-777 treatment did not reduce the acute mortality of the rats after CLP (Fig. 3A). As an assessment of the rats' health, we monitored changes in each animal's weight. Compared with the body weight of the sham group, the body weight of the CLP group decreased significantly over the first two days after surgery and then began to increase slowly. 
Unfortunately, compared with the vehicle group, the CLP + INT-777 group did not show a significantly different body weight (Fig. B). However, in terms of the clinical scores, the results were encouraging. Although there was no difference in clinical scores between the CLP + vehicle group and the CLP + INT777 group at 6 hours after CLP, clinical scores in the CLP + INT-777 group had significantly improved at 24 hours after CLP. Fig. C)

\subsection{Effects of INT-777 treatment on neuronal degeneration and cognitive impairment after CLP}

Nissl staining of hippocampus on day 19 after CLP. Within the ipsilateral CA1 region, neuronal loss and atrophy patterns were significantly greater in the CLP + vehicle group than in the Sham group. Compared to Vehicle group, INT - 777 treatment significantly reversed this neuronal damage in the ipsilateral CA1 region (Fig. 4A). In the Morris water maze test, compared to control rats, sepsis survivors were found to spend significantly more time on the platform, swim longer, and spend less time in the target quadrant after the platform was removed. Compared with the CLP + vehicle group, the treatment group showed a shorter escape latency time and swimming distance, and the duration that the rats remained in the target quadrant on the 4th and 5th days was longer in the treatment group (Fig. 4B-E). These results indicate that cognitive decline occurred in the rats after sepsis, but this decline could be prevented to some extent by INT-777 therapy. In the elevated plus-maze experiment, rats in the CLP + vehicle group took longer to enter the open arm, but INT-777 treatment significantly decreased this time (Fig. 4F), suggesting that TGR5 activation can reduce anxiety-like behavior.

\subsection{Effects of INT-777 on neuroinflammation after CLP}

To assess microglial/macrophage activation, neutrophil infiltration and inflammatory factor release, immunofluorescence staining was used to detect Iba-1, MPO and IL-1 $\beta$ levels in the hippocampus 24 hours after CLP. Compared with the CLP + vehicle group, the CLP + INT-777 group showed significantly fewer IBA-1-, MPO- and IL-1 $\beta$-positive cells in the hippocampus (Fig. 5A-D). For further quantitative analysis, western blot analysis was used to detect expression changes in IBA-1, MPO and IL-1 $\beta$ in the hippocampus of rats, and the results were consistent with those of immunofluorescence analysis. After CLP, the expression of IBA-1, MPO and IL-1 $\beta$ was significantly increased, but expression of these proteins was significantly decreased after INT-777 treatment (Fig. 5E-J).

\subsection{Effects of TGR5 CRISPR on neuroinflammation and signaling pathways at 24 hours after CLP}

To assess the role of TGR5 in neuroinflammation after CLP, endogenous TGR5 was knocked out by i.c.V administration of TGR5 CRISPR oligonucleotide. Western blot analysis showed that the expression levels of TGR5, cAMP, p-PKA, p-CREB, IL-1 $\beta$, IL- 6 and TNF-a were significantly increased after CLP. Compared with the CLP + vehicle group, the CLP + INT-777 group showed slightly increased TGR4 expression levels; 
significantly increased cAMP, p-PKA and p-CREB levels; and significantly decreased levels of the inflammatory cytokines IL-1 $\beta$, IL- 6 and TNF-a, indicating that INT-777 treatment had a significant inhibitory effect on neuroinflammation. When TGR5 knockdown was carried out via CRISPR, TGR5 expression in the hippocampus was significantly decreased. Compared with the CLP + INT-777 + scrambled CRISPR group, the CLP + INT-777 + TGR5 CRISPR group exhibited obviously decreased cAMP, p-PKA, p-CREB, IL-1 $\beta$, IL-6 and TNF-a expression levels, indicating that TGR5 knockout via CRISPR offset the positive effect of INT-777 in inhibiting neuroinflammation (Fig. 6A-D).

\subsection{Verification of the role of the CAMP/PKA/CREB signaling pathway in the inhibition of neuroinflammation by TGR5 activation}

To further evaluate the mechanism by which INT-777 inhibits neuroinflammation, rats were administered (i.c.v.) SQ22536, an inhibitor of CAMP. SQ22536 did not change TGR5 protein expression, but cAMP, pPKA and p-CREB expression was significantly decreased, accompanied by increased IL-1 $\beta$, IL- 6 and TNF-a expression, after SQ22536 administration. These results indicated that SQ22536 abolished the neuroprotective effects of INT-777 and also confirmed that the CAMP/PKA/CREB pathway is a possible mechanism by which INT-777 exerts a neuroprotective effect after CLP (Fig. 7A-D).

\section{Discussion}

In this study, we first demonstrated the role of TGR5 activation in neuroinflammation and long-term cognitive impairment after CLP in rats and that its effects may occur via the TGR5/CAMP/PKA/CREB signaling pathway. We found the following: 1) The expression of TGR5 in the hippocampus increased in a time-dependent manner and peaked at $3 \mathrm{~d}$ after CLP. The results of immunofluorescence analysis showed that TGR5 was expressed in microglial cells. 2) Although the intranasal administration of INT777, a selective inhibitor of TGR5, at 1 hour after CLP did not improve the survival and change in body weight of the rats, it reduced hippocampal microglial activation, neutrophil infiltration, and the expression of IL-1 $\beta$ in the hippocampus; it also reduced the clinical score 6 hours after surgery and improved spatial memory and anxiety-like behavior at 15 days. 3) Intracerebroventricular administration of TGR5 CRISPR and SQ22536 offset the protective effect of INT-777. These observations suggest that TGR activation inhibits neuroinflammation, at least in part, through the CAMP/PKA/CREB signaling pathway, thereby improving long-term cognitive impairment after CLP. INT-777, a selective inhibitor of TGR5, improved cognitive impairment and anxiety-like behavior in CLP rats.

Sepsis-associated encephalopathy (SAE) is the most common cause of encephalopathy in ICUs[4]. After discharge, up to $50 \%$ of sepsis survivors develop cognitive impairment that affects memory, attention, verbal fluency and executive function[3,26]. In addition, sepsis patients exhibit a higher incidence of mental illnesses, such as depression, anxiety and self-harm, than the general population[27]. Therefore, neurological dysfunction severely affects the quality of life of sepsis survivors and imposes a huge economic and social burden. In animal models of sepsis, surviving animals showed the same cognitive impairments and mental disorders shown by sepsis survivors[8, 28, 29]. In our study, we observed the 
same phenomenon. The Morris water maze test can be altered in numerous ways to investigate working memory, reference memory and task strategy[30]. The sepsis survivors in our study were found to spend significantly more time on the platform, swim longer, and spend less time in the target quadrant after the platform was removed. An elevated-plus maze experiment assessed anxiety or the fear of an open space in the rats; normally, animals consciously avoid the open arms and stay in a closed arm[31]. In our experiment, CLP rats took more time to enter an open arm. Not only the Morris water maze experiment but also the elevated plus-maze experiment shows the function of the hippocampus and prefrontal cortex in animals[32, 33]. Therefore, to further explore the mechanism by which INT-777 improves cognitive impairment in rats after CLP, we selected hippocampal tissue for further study.

The underlying pathologic changes in brain injury after sepsis are multifactorial and highly complex. Inflammatory cytokines, BBB destruction, ischemic processes, neurotransmitter changes, and mitochondrial dysfunction may be involved in this complex process, but its specific mechanisms have not yet been established[34]. However, uncontrolled neuroinflammation and ischemic injury are closely related to brain injury after sepsis[35]. Neuroinflammation is a major component of the pathology and progression of many neurological and neurodegenerative diseases[36]. Uncontrolled inflammation is a major cause of the abnormal function of brain cells (including microglia, neurons, and endothelial cells) and their increased apoptosis [37]. Microglial cells are the main macrophage cells in the brain, representing most of the brain immune system. Overactivation of microglial cells releases various harmful inflammatory mediators and increases the release of reactive oxygen species by destroying the stability of the blood-brain barrier, thus exacerbating brain dysfunction[38]. Indeed, studies have shown that inhibiting microglia helps reduce neuroinflammation in sepsis, which in turn improves long-term cognitive function[39]. Our results showed that the number of microglial cells in the hippocampus of rats at 24 hours after CLP was significantly increased compared to that in rats in the sham group, and MPO staining suggested that neutrophil infiltration was increased, with the inflammatory mediator IL-1 $\beta$ showed the same trend. The results of western blot analysis performed for further quantitative analysis also indicated that the protein expression levels of Iba- 1 , MPO and IL-1 $\beta$ in the hippocampus after CLP were significantly increased. Combined with the results of other studies, these results show that inhibition of early neuroinflammation after CLP may be an effective strategy to improve later cognitive dysfunction.

TGR5 (also called G-protein-coupled bile acid receptor, Gpbar1) is a member of the GPCR superfamily[9]. Surprisingly, numerous studies have shown that TGR agonists may be agents for the treatment of metabolic, inflammatory and digestive diseases[40]. In neurological diseases, activation of TGR5 by a different agonist also shows neuroprotective effects. McMillin et al. found that activating TGR5 could alleviate neuroinflammation and improve outcomes through neuron and microglia paracrine signaling in a mouse model of hepatic encephalopathy[15]. Lewis et al. also found that clinical scores in experimental autoimmune encephalitis model mice were significantly reduced after treatment with TGR5 agonists, which was associated with the reduced activation of monocytes and microglia cells[41]. 6a-Ethyl-23(S)methylcholic acid (6-EMCA, INT-777) is a selective, specific agonist for TGR5, and Zuo et al. found that intranasal administration of INT-777 was also beneficial for SAH rats[12]. In our study, after the intranasal administration of INT-777 one hour after CLP, cognitive impairment and anxiety-like behavior in CLP rats 
significantly improved and the number of activated microglia and neutrophil infiltration were significantly reduced, and the results of WB analysis showed that inflammatory factors were also downregulated. After TGR5 CRISPR-mediated knockout of TGR5 in CLP animals, the neuroprotective effect of INT-777 disappeared. All these results suggest that INT-777 can inhibit microglial activation and inflammation by activating TGR5, thereby improving long-term cognitive impairment.

G protein coupled receptors (GPCRs) are thought to play a role in signal transduction by producing a number of second messengers, including CAMP. When activated by INT-777, TGR5 leads to increased intracellular cAMP levels and activates subsequent signaling pathways[42]. Studies have shown that the TGR5-cAMP-PKA axis is the basis of bile acid-mediated anti-inflammatory effects[19]. PKA promotes phosphorylation of the transcriptional activation sites of CREB, which further promotes the binding of CREB to the transcriptional coactivator CBP and the formation of complexes that block the transcription of inflammatory genes[43]. CREB signaling also reduces the activation of proinflammatory microglial cells, leading to neuroprotective effects[44].

In the current study, we found that INT-777 increased the expression of CAMP/P-PKA/P-CREB after CLP, while CRISPR-mediated TGR5 knockout inhibited the expression of these proteins. To further confirm our hypothesis, SQ22536, an adenylyl cyclase inhibitor, was used to inhibit CAMP, and the results showed that the expression of TGR5 was not changed, but the expression of cAMP/p-PKA/p-CREB was decreased, and expression of the inflammatory cytokines IL-1 $\beta$, IL- 6 and TNF- $a$ was significantly increased, suggesting that the neuroprotective effect of TGR5 had been reversed by SQ22536. These findings support the idea that the improvement in cognitive impairment observed in rats after CLP with INT-777 treatment is achieved through the TGR5/CAMP/PKA/CREB signaling pathway.

This study has some limitations. First, although TGR5 plays a role in neuroprotection through different mechanisms, such as resistance to apoptosis, oxidative stress resistance, and the maintenance of bloodbrain barrier integrity, in this study, the role of TGR5 in only neuroinflammation was studied. Second, our study showed that TGR5 is also minimally expressed on astrocytes, and the current study confirms that astrocytes are also strongly associated with neuroinflammation[45], and we intend to further evaluate the effect of INT-777 treatment on astrocytes in neuroinflammation in a follow-up study. Third, INT-777 was administered at 1 hour after CLP, and whether the administration of INT-777 within a time frame of 3 hours, 6 hours or longer after CLP would still have a significant neuroprotective effect was not evaluated, although this information would be more valuable for clinical application.

\section{Conclusions}

Experiments in surviving sepsis rats showed that INT-777 treatment reduced neuroinflammation and microglial cell activation and improved cognition and behavior. If translated into clinical practice, TGR5 may be a therapeutic target to improve neurological outcomes in sepsis survivors.

\section{Abbreviations}


SAE

sepsis- associated encephalopathy;

BBB

blood-brain barrier; Gpbar1/TGR5:G-protein-coupled bile acid receptor; cAMP:cyclic adenosine monophosphate; PKA:protein kinase A; CREB:cAMP response element-binding protein; INT-777:6R-ethyl23(S)-methylcholic acid; CLP:cecal ligation and puncture; DAPI:4',6-diamidino-2- phenylindole; Iba-

1:ionized calcium-binding adaptor molecule-1; MPO:Myeloperoxidase

\section{Declarations}

\section{Ethical Approval and Consent to Participate}

All experimental protocols were approved by the Institutional Animal Care and Use Committee (IACUC) of Fudan University, which comply with the National Institutes of Health Guidelines for the Care and Use of laboratory Animals in Neuroscience Research and ARRIVE guidelines.

\section{Consent for publication}

Not applicable.

\section{Competing interest}

The authors declare that they have no conflict of interest.

\section{Data availability statement}

The data support the findings of this study and are available from the corresponding author upon reasonable request.

\section{Authors' contributions}

This study was designed by PJ, SXD, YG. The experiments were completed by PJ, SXD, MT, PJW, YW, HMW, JYT and FZ. PJ, SXD, and MT performed the statistical analysis. PJ finished writing the manuscript. YQG and YG provided supervision and final check. All the authors read the final version of this paper and approved it.

\section{Funding}

This study was supported by grants from the National Key R\&D Program of China (2018YFC1312600, 2018YFC1312604 to Y. G.), the National Natural Foundation of China (81772674 to Y.G.), the Science and Technology Commission of Shanghai Municipal (18140901100 to Y.G.), Shanghai Municipal Health Commission, Health Sector Clinical Research Special Youth Project (20184Y0064 to S.D. 20194 Y0444 to M.T.) 
Acknowledgements

Not applicable.

\section{Authors' information}

'Department of Intensive Care Unit, HuaShan Hospital, Fudan University, Shanghai, 200040, China.

${ }^{2}$ Department of Neurosurgery, Huashan Hospital, Fudan University, Shanghai, 200040, China.

${ }^{3}$ State Key Laboratory of Medical Neurobiology and MOE Frontiers Center for Brain Science, Institutes of Brain Science, Fudan University, Shanghai, 200032, China

\section{References}

1. Angus DC, Linde-Zwirble WT, Lidicker J, Clermont G, Carcillo J, Pinsky MR. Epidemiology of severe sepsis in the United States: analysis of incidence, outcome, and associated costs of care. Crit Care Med. 2001;29:1303-10.

2. Annane D, Sharshar T. Cognitive decline after sepsis. Lancet Respir Med. 2015;3:61-9.

3. Iwashyna TJ, Ely EW, Smith DM, Langa KM. Long-term cognitive impairment and functional disability among survivors of severe sepsis. JAMA. 2010;304:1787-94.

4. Gofton TE, Young GB. Sepsis-associated encephalopathy. Nat Rev Neurol. 2012;8:557-66.

5. Chaudhry N, Duggal AK. Sepsis Associated Encephalopathy. Adv Med. 2014;2014:762320.

6. Savio LEB, Andrade MGJ, de Andrade Mello P, Santana PT, Moreira-Souza ACA, Kolling J, Longoni A, Feldbrugge L, Wu Y, Wyse ATS, et al. P2 × 7 Receptor Signaling Contributes to Sepsis-Associated Brain Dysfunction. Mol Neurobiol. 2017;54:6459-70.

7. Schwalm MT, Pasquali M, Miguel SP, Dos Santos JP, Vuolo F, Comim CM, Petronilho F, Quevedo J, Gelain DP, Moreira JC, et al. Acute brain inflammation and oxidative damage are related to long-term cognitive deficits and markers of neurodegeneration in sepsis-survivor rats. Mol Neurobiol. 2014;49:380-5.

8. Silva AYO, Amorim EA, Barbosa-Silva MC, Lima MN, Oliveira HA, Granja MG, Oliveira KS, Fagundes PM, Neris RLS, Campos RMP, et al: Mesenchymal Stromal Cells Protect the Blood-Brain Barrier, Reduce Astrogliosis, and Prevent Cognitive and Behavioral Alterations in Surviving Septic Mice. Crit Care Med 2020.

9. Guo C, Chen WD, Wang YD. TGR5, Not Only a Metabolic Regulator. Front Physiol. 2016;7:646.

10. Tiwari A, Maiti P. TGR5: an emerging bile acid G-protein-coupled receptor target for the potential treatment of metabolic disorders. Drug Discov Today. 2009;14:523-30.

11. Liang H, Matei N, McBride DW, Xu Y, Tang J, Luo B, Zhang JH. Activation of TGR5 protects blood brain barrier via the BRCA1/Sirt1 pathway after middle cerebral artery occlusion in rats. J Biomed Sci. 2020;27:61. 
12. Zuo G, Zhang T, Huang L, Araujo C, Peng J, Travis Z, Okada T, Ocak U, Zhang G, Tang J, et al. Activation of TGR5 with INT-777 attenuates oxidative stress and neuronal apoptosis via cAMP/PKCepsilon/ALDH2 pathway after subarachnoid hemorrhage in rats. Free Radic Biol Med. 2019;143:441-53.

13. Duboc H, Tache $Y$, Hofmann AF. The bile acid TGR5 membrane receptor: from basic research to clinical application. Dig Liver Dis. 2014;46:302-12.

14. Wang XX, Edelstein MH, Gafter U, Qiu L, Luo Y, Dobrinskikh E, Lucia S, Adorini L, D'Agati VD, Levi J, et al. G Protein-Coupled Bile Acid Receptor TGR5 Activation Inhibits Kidney Disease in Obesity and Diabetes. J Am Soc Nephrol. 2016;27:1362-78.

15. McMillin M, Frampton G, Tobin R, Dusio G, Smith J, Shin H, Newell-Rogers K, Grant S, DeMorrow S. TGR5 signaling reduces neuroinflammation during hepatic encephalopathy. J Neurochem. 2015;135:565-76.

16. Wu X, Lv YG, Du YF, Hu M, Reed MN, Long Y, Suppiramaniam V, Hong H, Tang SS. Inhibitory effect of INT-777 on lipopolysaccharide-induced cognitive impairment, neuroinflammation, apoptosis, and synaptic dysfunction in mice. Prog Neuropsychopharmacol Biol Psychiatry. 2019;88:360-74.

17. Kawamata Y, Fujii R, Hosoya M, Harada M, Yoshida H, Miwa M, Fukusumi S, Habata Y, Itoh T, Shintani Y, et al. A G protein-coupled receptor responsive to bile acids. J Biol Chem. 2003;278:943540.

18. Kumar DP, Rajagopal S, Mahavadi S, Mirshahi F, Grider JR, Murthy KS, Sanyal AJ. Activation of transmembrane bile acid receptor TGR5 stimulates insulin secretion in pancreatic beta cells. Biochem Biophys Res Commun. 2012;427:600-5.

19. Guo C, Xie S, Chi Z, Zhang J, Liu Y, Zhang L, Zheng M, Zhang X, Xia D, Ke Y, et al. Bile Acids Control Inflammation and Metabolic Disorder through Inhibition of NLRP3 Inflammasome. Immunity. 2016;45:944.

20. Wu D, Zhang S, Sun N, Zhu B, Lin S. Neuroprotective Function of a Novel Hexapeptide QMDDQ from Shrimp via Activation of the PKA/CREB/BNDF Signaling Pathway and Its Structure-Activity Relationship. J Agric Food Chem 2020.

21. Rittirsch D, Huber-Lang MS, Flierl MA, Ward PA. Immunodesign of experimental sepsis by cecal ligation and puncture. Nat Protoc. 2009;4:31-6.

22. Bobeck EN, Chen Q, Morgan MM, Ingram SL. Contribution of adenylyl cyclase modulation of pre- and postsynaptic GABA neurotransmission to morphine antinociception and tolerance. Neuropsychopharmacology. 2014;39:2142-52.

23. Rogers DC, Fisher EM, Brown SD, Peters J, Hunter AJ, Martin JE. Behavioral and functional analysis of mouse phenotype: SHIRPA, a proposed protocol for comprehensive phenotype assessment. Mamm Genome. 1997;8:711-3.

24. Vorhees CV, Williams MT. Morris water maze: procedures for assessing spatial and related forms of learning and memory. Nat Protoc. 2006;1:848-58. 
25. Wang T, Zhang J, Li P, Ding Y, Tang J, Chen G, Zhang JH. NT-4 attenuates neuroinflammation via TrkB/PI3K/Fox01 pathway after germinal matrix hemorrhage in neonatal rats. J Neuroinflammation. 2020;17:158.

26. Iwashyna TJ, Speelmon EC. Advancing a Third Revolution in Critical Care. Am J Respir Crit Care Med. 2016;194:782-3.

27. Wintermann GB, Brunkhorst FM, Petrowski K, Strauss B, Oehmichen F, Pohl M, Rosendahl J. Stress disorders following prolonged critical illness in survivors of severe sepsis. Crit Care Med. 2015;43:1213-22.

28. Reis PA, Alexandre PCB, D'Avila JC, Siqueira LD, Antunes B, Estato V, Tibirica EV, Verdonk F, Sharshar $\mathrm{T}$, Chretien $\mathrm{F}$, et al. Statins prevent cognitive impairment after sepsis by reverting neuroinflammation, and microcirculatory/endothelial dysfunction. Brain Behav Immun. 2017;60:293-303.

29. Barichello T, Machado RA, Constantino L, Valvassori SS, Reus GZ, Martins MR, Petronilho F, Ritter C, Quevedo J, Dal-Pizzol F. Antioxidant treatment prevented late memory impairment in an animal model of sepsis. Crit Care Med. 2007;35:2186-90.

30. Nunez J. Morris Water Maze Experiment. J Vis Exp 2008.

31. Pellow S, Chopin P, File SE, Briley M. Validation of open:closed arm entries in an elevated plus-maze as a measure of anxiety in the rat. J Neurosci Methods. 1985;14:149-67.

32. Moser El, Krobert KA, Moser MB, Morris RG. Impaired spatial learning after saturation of long-term potentiation. Science. 1998;281:2038-42.

33. D'Hooge R, De Deyn PP. Applications of the Morris water maze in the study of learning and memory. Brain Res Brain Res Rev. 2001;36:60-90.

34. Adam N, Kandelman S, Mantz J, Chretien F, Sharshar T. Sepsis-induced brain dysfunction. Expert Rev Anti Infect Ther. 2013;11:211-21.

35. Ren C, Yao RQ, Zhang H, Feng YW, Yao YM. Sepsis-associated encephalopathy: a vicious cycle of immunosuppression. J Neuroinflammation. 2020;17:14.

36. Meneses G, Cardenas G, Espinosa A, Rassy D, Perez-Osorio IN, Barcena B, Fleury A, Besedovsky H, Fragoso G, Sciutto E. Sepsis: developing new alternatives to reduce neuroinflammation and attenuate brain injury. Ann N Y Acad Sci. 2019;1437:43-56.

37. Dal-Pizzol F, Tomasi CD, Ritter C. Septic encephalopathy: does inflammation drive the brain crazy? Braz J Psychiatry. 2014;36:251-8.

38. Michels M, Danielski LG, Dal-Pizzol F, Petronilho F. Neuroinflammation: microglial activation during sepsis. Curr Neurovasc Res. 2014;11:262-70.

39. Michels M, Vieira AS, Vuolo F, Zapelini HG, Mendonca B, Mina F, Dominguini D, Steckert A, Schuck PF, Quevedo J, et al. The role of microglia activation in the development of sepsis-induced long-term cognitive impairment. Brain Behav Immun. 2015;43:54-9.

40. Broeders EP, Nascimento EB, Havekes B, Brans B, Roumans KH, Tailleux A, Schaart G, Kouach M, Charton J, Deprez B, et al. The Bile Acid Chenodeoxycholic Acid Increases Human Brown Adipose 
Tissue Activity. Cell Metab. 2015;22:418-26.

41. Lewis ND, Patnaude LA, Pelletier J, Souza DJ, Lukas SM, King FJ, Hill JD, Stefanopoulos DE, Ryan K, Desai S, et al. A GPBAR1 (TGR5) small molecule agonist shows specific inhibitory effects on myeloid cell activation in vitro and reduces experimental autoimmune encephalitis (EAE) in vivo. PLoS One. 2014;9:e100883.

42. Watanabe M, Houten SM, Mataki C, Christoffolete MA, Kim BW, Sato H, Messaddeq N, Harney JW, Ezaki $\mathrm{O}$, Kodama $\mathrm{T}$, et al. Bile acids induce energy expenditure by promoting intracellular thyroid hormone activation. Nature. 2006;439:484-9.

43. Xie W, Li F, Han Y, Qin Y, Wang Y, Chi X, Xiao J, Li Z. Neuropeptide Y1 receptor antagonist promotes osteoporosis and microdamage repair and enhances osteogenic differentiation of bone marrow stem cells via cAMP/PKA/CREB pathway. Aging. 2020;12:8120-36.

44. Bhat SA, Henry RJ, Blanchard AC, Stoica BA, Loane DJ, Faden Al. Enhanced Akt/GSK-3beta/CREB signaling mediates the anti-inflammatory actions of mGluR5 positive allosteric modulators in microglia and following traumatic brain injury in male mice. J Neurochem 2020:e14954.

45. Tschoe C, Bushnell CD, Duncan PW, Alexander-Miller MA, Wolfe SQ. Neuroinflammation after Intracerebral Hemorrhage and Potential Therapeutic Targets. J Stroke. 2020;22:29-46.

\section{Figures}

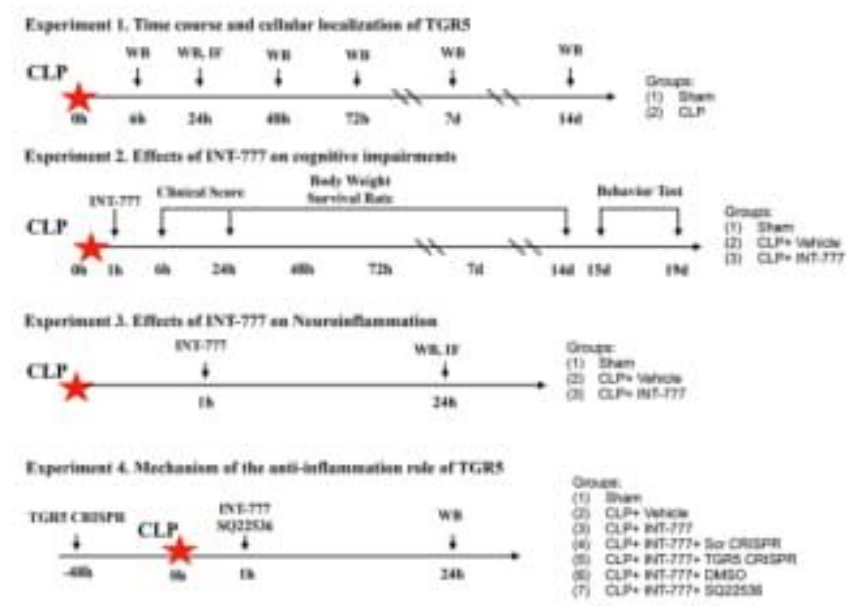

\section{Figure 1}

Experimental design and animal group classification. CLP, Cecal Ligation and Puncture; WB, western blot; IF, immunofluorescence. 

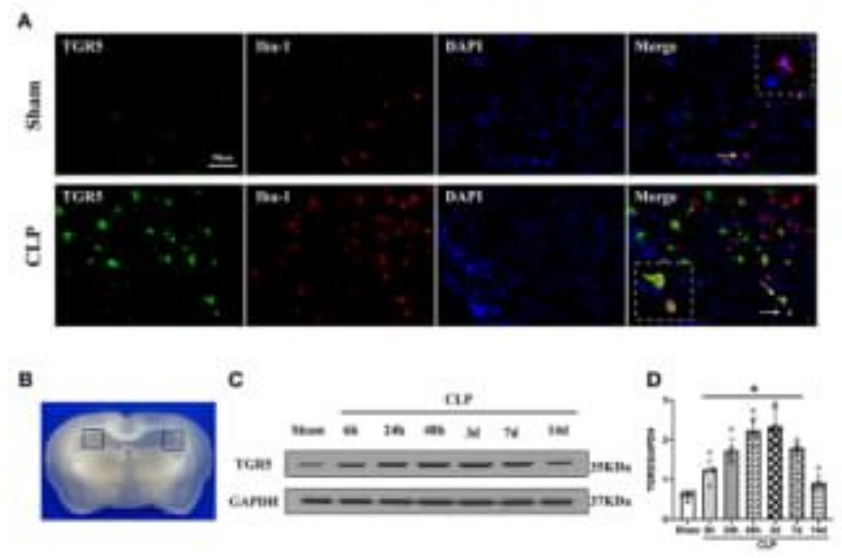

\section{Figure 2}

Time course and cellular localization of endogenous TGR5 expression in hippocampus after CLP. (A) Double immunofluorescence staining for TGR5 (green) in microglia (Iba-1, red), $n=2 /$ group, Scale bar = $50 \mu \mathrm{m}$. (DAPI, 4',6-diamidino-2-phenylindole; Iba-1, ionized calcium-binding adaptor molecule-1.) (B) Schematic diagram of brain tissue, with the hippocampal area marked in black; The images were taken out for immunofluorescence staining. (C) Representative Western Blot images and (D) quantitative analyses of TGR5 time course after CLP, $n=6 /$ group, ${ }^{\star} p<0.05$ vs indicated groups. Bars represent mean \pm SD.
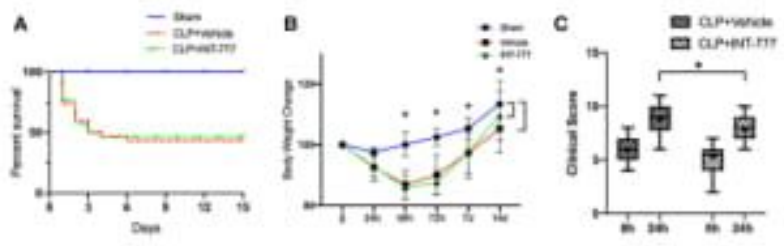

\section{Figure 3}

INT-777 effects on survival, clinical score, and body weight change after CLP. (A) Percentage of surviving animals each group 15 days after CLP, (B) Body weight gain in each group, $n=10-21 /$ group, (C) Clinical score assessed 6 hours and 24 hours after CLP, ${ }^{\star} p<0.05$ vs indicated groups, bars represent mean \pm SD. 

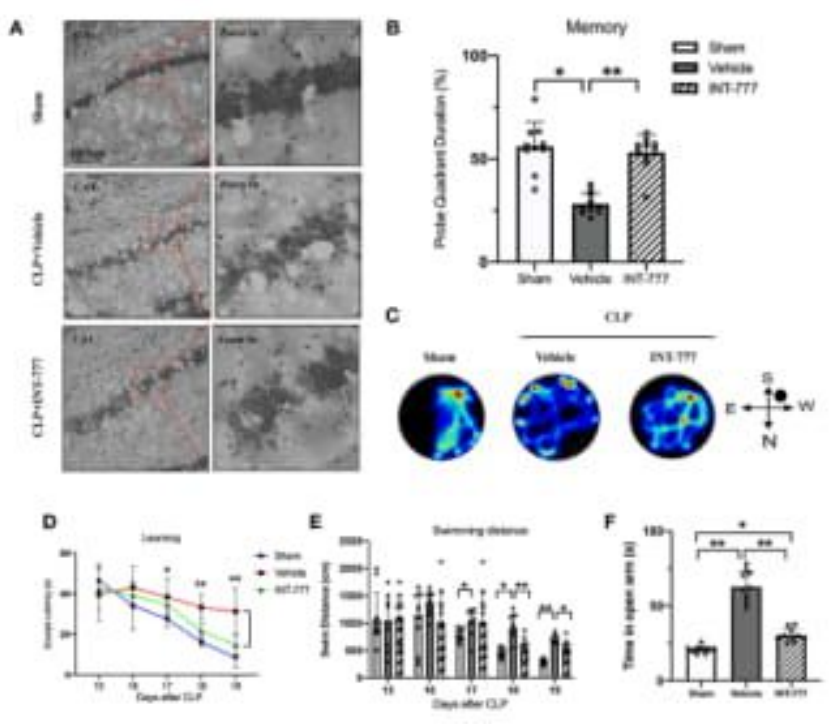

Figure 4

Effects of INT-777 treatment on neuronal degeneration and cognitive impairment after CLP. (A) Representative micrographs and neuronal quantifications of Nissl staining in hippocampal CA1 region, (B) Probe quadrant duration, (C) Representative heat map in probe test, (D) Escape latency, (E) Swim distance of Morris water maze on 15-19 days after CLP, (F) Time in open arm during the elevated plus maze test. $n=10-21 /$ group, ${ }^{*} p<0.05$ and ${ }^{* *} p<0.01$ vs indicated groups, bars are represented as mean \pm SD.
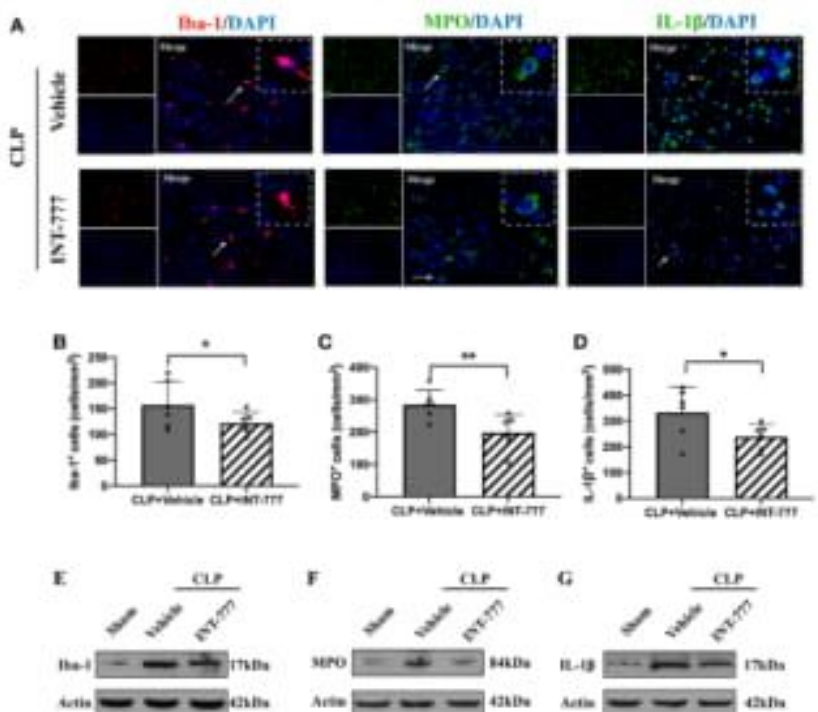

II
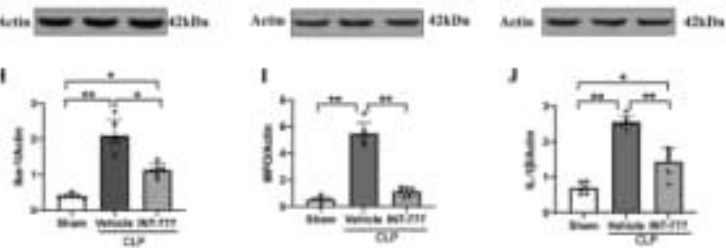

Figure 5

Effects of INT-777 on microglia/ macrophage activation and neuroinflammation after CLP. (A) Representative images of immunofluorescence staining of Iba-1 (red), MPO (green) and IL-1 $\beta$ (green) in 
the hippocampus area 24 hours after CLP, (B, C, D) Quantitative analyses of Iba-1, MPO, and IL-1 $\beta$-positive cells in the hippocampus area, $(E, F, G)$ Representative Western blot images and $(H, I, J)$ quantitative analyses of Iba-1, MPO and IL-1 $\beta$ levels in the hippocampus 24 hours after CLP. $n=6 /$ group, ${ }^{*} p<0.05$ and ${ }^{* *} p<0.01$ vs indicated groups, bars are represented as mean \pm SD.

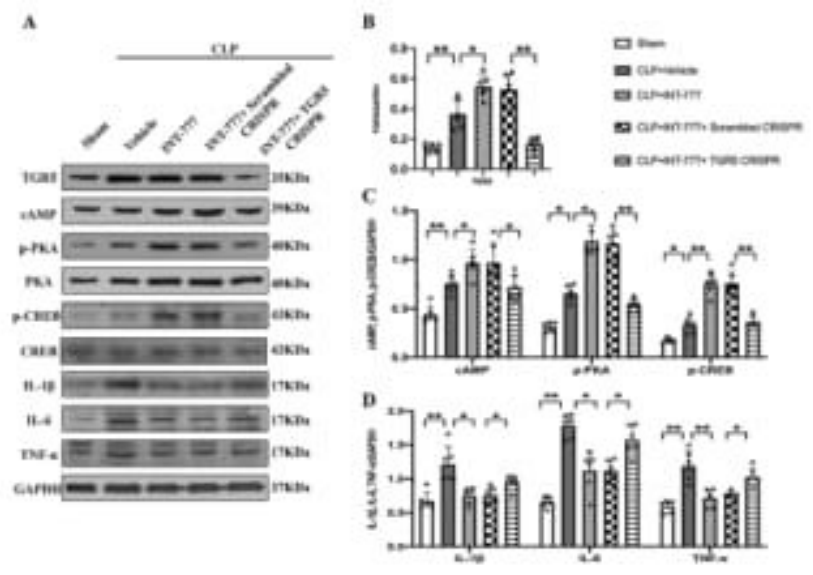

Figure 6

Effects of TGR5 CRISPR on neuroinflammation and signaling pathway at 24 hours after CLP, (A) Representative Western blot images and (B - D) Quantitative analyses of TGR5, cAMP, p-PKA, p-CREB, IL$1 \beta$, IL- 6 and TNF- $\alpha$ in the hippocampus area at $24 \mathrm{~h}$ after CLP. $n=6$ /group, ${ }^{*}<0.05$ and ${ }^{*} p<0.01$ vs indicated groups, bars are represented as mean \pm SD.

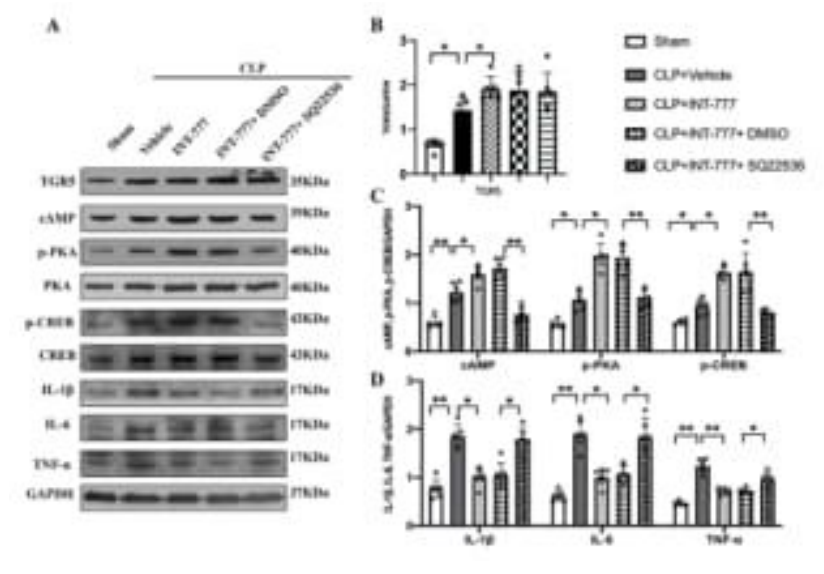

Figure 7

Effects of SQ22536 on signaling pathway at 24 hours after CLP, (A) Representative Western blot images and (B - D) Quantitative analyses of TGR5, cAMP, p-PKA, p-CREB, IL-1 $\beta$, IL- 6 and TNF- $\alpha$ in the hippocampus area at $24 \mathrm{~h}$ after CLP. $n=6 /$ group, ${ }^{*} p<0.05$ and ${ }^{* *} p<0.01$ vs indicated groups, bars are represented as mean \pm SD.

\section{Supplementary Files}

This is a list of supplementary files associated with this preprint. Click to download. 
- Supplementaryfigure.docx

- SupplementaryFigures.tif 